\title{
Expression of DNA methyltransferases and target microRNAs in human tissue samples related to sporadic colorectal cancer
}

\author{
CHAO HUANG, HONG LIU, XIU-LI GONG, LIYUN WU and BIN WEN \\ PI-WEI Institute of Guangzhou University of Chinese Medicine, Guangzhou, Guangdong 510000, P.R. China
}

Received March 13, 2016; Accepted July 19, 2016

DOI: $10.3892 /$ or.2016.5104

\begin{abstract}
Tissue microenvironment functions as a pivotal mediator in colorectal carcinogenesis, and its alteration can cause some important cellular responses including epigenetic events. The present study examined histologically altered tissue structure, DNA methyltransferases (DNMTs) and their corresponding expression of target microRNAs (miRNA). Tissues resected by surgery were from primary colorectal carcinoma. These samples were from three locations: and were $\geq 10,5$ and $\leq 2 \mathrm{~cm}$ away from the proximal lesion of colon cancer, and marked as no. 1, no. 2 and no. 3, respectively. Histological alteration was assessed by H\&E staining, expression of DNMT1, DNMT3A, and DNMT3B was detected by immunohistochemistry and western blotting, microarray chip was used to screen distinguishable miRNAs and miRNAs targeting DNMTs whose validation assay was performed by quantitative real-time polymerase chain reaction (qRT-PCR). Our results revealed that normal crypt structure was shown in no. 1, while many aberrant crypt foci appeared in no. 3. Significant upregulation of DNMT1, DNMT3A, and DNMT3B expression was found in para-carcinoma tissues, compared with the histopathologically unchanged tissues $(\mathrm{P}<0.05)$, furthermore, distinguishable expression profiling was observed of target miRNAs in tissues with different distance. Our results provide additional insights for future research of colorectal carcinogenesis by introducing the tissue microenvironment.
\end{abstract}

\section{Introduction}

Colorectal cancer (CRC) with a multistep process is a leading cause of high cancer-related morbidity and mortality, with more than 600,000 deaths worldwide annually (1). Although various etiologies, which have not been completely established exist, the aberrant accumulation of genetic and epigenetic

Correspondence to: Professor Bin Wen, PI-WEI Institute of Guangzhou University of Chinese Medicine, Guangzhou, Guangdong 510000, P.R. China

E-mail: wenbin@gzucm.edu.cn

Key words: tumor microenvironment, colorectal cancer, DNA methyltransferases, microRNAs, epigenetics changes remains one of the important mechanisms. Similar to genetic lesions, epigenetic lesions can also change structure and function of genome without the alteration of DNA nucleotide sequence (2), and present heritable and possibly reversible changes $(2,3)$. Recently, numerous data have shown that alteration of epigenetic modification involving DNA methylation, histone modifications, miRNAs and long non-coding RNAs contribute to carcinogenesis (4), suggesting that DNA methylation changes may create a carcinogenic field in histologically normal mucosa of CRC patients. In this setting, an opportunity to study the environmental effects on epigenetic alteration reveals a novel challenge to better clarify the function of epigenetics on CRC tumorigenesis (5). DNA methylation profiles are regulated by DNA methyltransferases (DNMTs), hence, an aberrant DNMT expression results in abnormal DNA methylation status $(6,7)$. In addition, upregulated expression of DNMTs is often prior to change of hypermethylation in gene promoter, which is considered to be an early molecular hallmark of cancer cells (7). Therefore, in this context, our research group performed this study for the first time, we presented a hypothesis that tissues with different distance from CRC focus display varied microenvironment playing an important role in CRC carcinogenesis, and epigenetic alteration is a crucial player in cellular response to the changed microenvironment.

Recently, miRNAs have attracted extensive attention owing to their important functions in a large number of biological activities, such as cellular differentiation, cell proliferation and apoptosis $(8,9)$. MicroRNAs (miRNAs or miR) are a class of endogenous small non-coding RNAs known to regulate gene expression at the post-transcriptional level through binding to a complementary site that locates on the 3'-untranslated region of target mRNAs (10). Dysregulation of miRNA expression is found in many forms of cancer, including esophageal squamous cell carcinoma (11), breast cancer (12), and gastric cancer (13). Although previous studies have manifested that global genome hypomethylation presented the characteristic of step-by-step wide genome depletion of methylated cytosine base pairs (5-methylated cytosine) (14), and a mass of aberrant miRNAs have been discovered in CRC as well (15), which seem to have shown the significant role of miRNAs in development of $\mathrm{CRC}$, the difference and relative function of the miRNAs targeting DNMTs in precancerous lesions of CRC are still unclear. Therefore, we performed the present study to elucidate the global changes of miRNA expression 
profiles and the expression difference of miRNAs targeting corresponding DNMTs in different locations with different distance from cancer lesions under the above hypothesis.

\section{Materials and methods}

Tissue samples and reagents. Tissues resected operationally were from the First Affiliated Hospital of Guangzhou University of Traditional Chinese Medicine (TCM). All samples were sporadic CRC, and were not administered any radiotherapy or chemotherapy before operation. The diagnosis of CRC were confirmed by at least two pathologists. Samples collected were immediately frozen in liquid nitrogen. Samples obtained from three sites from the same patient were, respectively, marked as no. 1 , no. 2 and no. 3 , which were $\geq 10,5$ and $\leq 2 \mathrm{~cm}$ away from the CRC lesions, respectively (as shown in Fig. 1A).

Reagents and instruments used in this study included TRIzol reagent and glycogen without RNaseA (Invitrogen Life Technologies, Carlsbad, CA, USA), RNase inhibitor, reverse transcriptase and 10X RT buffer solution $(250 \mathrm{mM}$ Tris-HCl, $\mathrm{pH} 8.3,200 \mathrm{mM} \mathrm{KCl}, 40 \mathrm{mM} \mathrm{MgCl} 2,5 \mathrm{mM}$ DTT) (all from Epicentre Biotechnologies, Madison, WI, USA), $2.5 \mathrm{mM}$ dNTP mixed liquids (dATP, dGTP, dCTP and dTTP $2.5 \mathrm{mM}$, respectively) (HyTest Ltd., Turku, Finland), RT primers (Invitrogen Life Technologies); 2X PCR Master mix (SuperArray Bioscience, Frederick, MD, USA), Primer 5.0 ABI Prism 7900 system (Applied Biosystems Inc., Foster City, CA, USA). TRIzol (Invitrogen) and miRNeasy mini kit (Qiagen, Hilden, Germany), miRCURY ${ }^{\mathrm{TM}}$ LNA array (v.18.0), Axon GenePix 4000B microarray scanner, GenePix Pro 6.0 (Axon Instruments, Foster City, CA, USA).

$H \& E$ staining for tissue structure. The tissues collected were fixed with $4 \%$ paraformaldehyde, and then paraffin-embedded samples were cut into $4 \mu \mathrm{m}$ slides. After a 10 -min and a 3 -min treatment of dimethylbenzene and ethanol, respectively, the slices were stained with hematoxylin for $10 \mathrm{~min}$ and subsequently $0.5 \%$ eosin for $3 \mathrm{~min}$, and then again treated with ethanol. The slices sealed with neutral gum were observed to obtain images using an inverted phase contrast microscope.

Immunohistochemical (IHC) analysis for biological markers and DNMTs. The $4 \mu \mathrm{m}$ thickness sections from the tissue block were selected for IHC staining. Briefly, samples which were fixed with $4 \%$ paraformaldehyde for 24 to $48 \mathrm{~h}$ were dehydrated, permeabilized and embedded in paraffin. Four micrometers of paraffin-embedded slides were deparaffized by dimethylbenzene for $10 \mathrm{~min}$ and incubated with $5 \%$ fetal bovine serum at room temperature for $10 \mathrm{~min}$. Antigen retrieval was performed separately using moderate heat-induced antigen retrieval two times in microwave oven. IHC was performed using rabbit anti-human CK18, CD133, vimentin, cyclin D1 (CD1) monoclonal antibodies. Primary antibodies were diluted in antibody diluent (Beyotime, Wuhan, China) to the indicated optimal dilutions of 1:100 for CK18; 1:100 for CD1; 1:50 for vimentin, 1:100 for CD133. The sections were incubated with a biotinylated goat anti-rabbit IgG secondary antibody. The slides were stained with diaminobenzidine (DAB) staining used as the chromogen for optimal time, then the sections were counterstained with hematoxylin. Optical density (OD) values of a positive immune product for each group were obtained by pathological analysis software IPP 6.0.

Western blotting. Total protein from tissue samples was extracted. Seperation of proteins $(30 \mu \mathrm{g})$ was performed by $10 \%$ sodium dodecyl sulphate-polyacrylamide gel electrophoresis (SDS-PAGE), and then these were transferred onto PVDF membrane (Millipore Corp., Billerica, MA, USA). After the membrane was incubated with rabbit anti-human DNMT1 antibody (bs-0678R), DNMT3A antibody (bs-0301R) and DNMT3B (bs-0301R) (all from BIOSS, Beijing, China) and $\beta$-actin antibody (ab129348; Abcam, San Francisco, CA, USA) at $4^{\circ} \mathrm{C}$ overnight, secondary antibodies were added to the membranes for further incubation. Detection of these proteins was performed by enhanced chemiluminescence. The quantification of images was measured using AS image analysis system (Kontron AG, Augsburg, Germany). Finally, the absolute values of the protein expression was quantified through the darkness of the DNMT1, DNMT3A, and DNMT3B bands divided by the darkness of $\beta$-actin bands.

miRNA microarray chip. The 7th generation of miRCURY ${ }^{\mathrm{TM}}$ LNA array (v.18.0) (Exiqon, Vedbaek, Denmark) contains 3,100 capture probes, covering all human, mouse and rat miRNAs annotated in miRBase 18.0 , as well as all viral miRNAs related to these species. In addition, this array contains capture probes for 25 miRPlus $^{\mathrm{TM}}$ human miRNAs. Total RNA was isolated using TRIzol (Invitrogen) and miRNeasy mini kit (Qiagen) according to manufacturer's instructions, which efficiently recovered all RNA species, including miRNAs. RNA quality and quantity was measured by using NanoDrop spectrophotometer (ND-1000; NanoDrop Technologies, Wilmington, DE, USA) and RNA integrity was determined by gel electrophoresis. After RNA isolation from the samples, the miRCURY ${ }^{\mathrm{TM}} \mathrm{Hy} 3^{\mathrm{TM}} / \mathrm{Hy} 5^{\mathrm{TM}}$ Power labeling kit (Exiqon) was used according to the manufacturer's guideline for miRNA labelling. One microgram of each sample was $3^{\prime}$-end-labeled with $\mathrm{Hy} 3^{\mathrm{TM}}$ fluorescent label, using T4 RNA ligase by the following procedure: RNA in $2.0 \mu \mathrm{l}$ of water was combined with $1.0 \mu \mathrm{l}$ of CIP buffer and CIP (Exiqon). The mixture was incubated for $30 \mathrm{~min}$ at $37^{\circ} \mathrm{C}$, and was terminated by incubation for $5 \mathrm{~min}$ at $95^{\circ} \mathrm{C}$. Then $3.0 \mu \mathrm{l}$ of labeling buffer, $1.5 \mu \mathrm{l}$ of fluorescent label (Hy3 ${ }^{\mathrm{TM}}$ ), $2.0 \mu \mathrm{l}$ of DMSO, $2.0 \mu \mathrm{l}$ of labeling enzyme were added into the mixture. The labeling reaction was incubated for $1 \mathrm{~h}$ at $16^{\circ} \mathrm{C}$, and terminated by incubation for $15 \mathrm{~min}$ at $65^{\circ} \mathrm{C}$.

Array hybridization. After stopping the labeling procedure, the $\mathrm{Hy} 3^{\mathrm{TM}}$-labeled samples were hybridized on the miRCURY'TM LNA array (v.18.0) (Exiqon) according to array manual. The total $25 \mu \mathrm{l}$ mixture from $\mathrm{Hy}^{\mathrm{TM}}$-labeled samples with $25 \mu 1$ hybridization buffer were first denatured for $2 \mathrm{~min}$ at $95^{\circ} \mathrm{C}$, incubated on ice for $2 \mathrm{~min}$ and then hybridized to the microarray for 16 to $20 \mathrm{~h}$ at $56^{\circ} \mathrm{C}$ in a 12 -Bay Hybridization Systems (Hybridization System-NimbleGen Systems, Inc., Madison, WI, USA), which provides an active mixing action and constant incubation temperature to improve hybridization uniformity and enhance signal. Following hybridization, the slides were achieved, washed several times using Wash buffer 


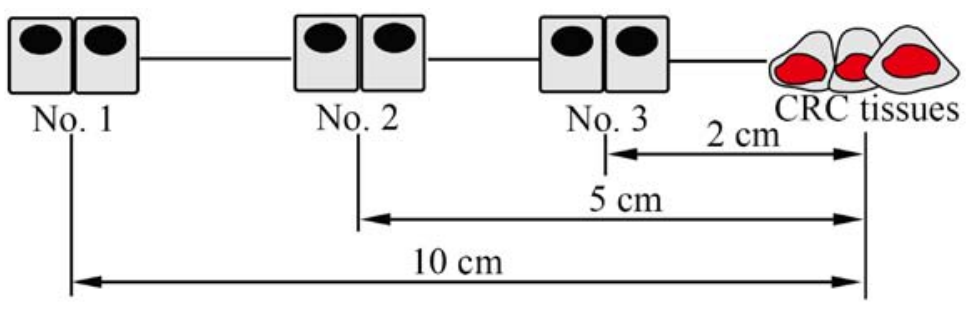

A
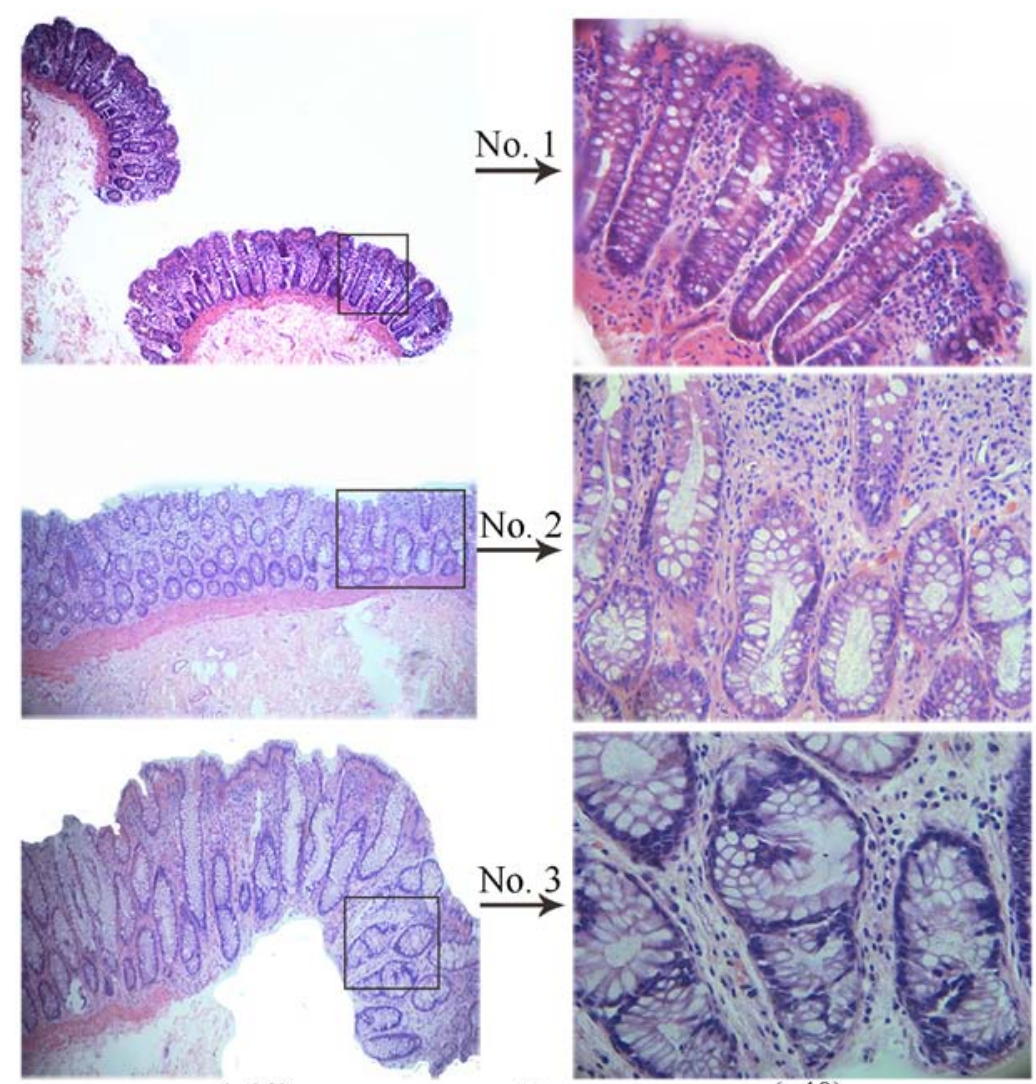

(x10)

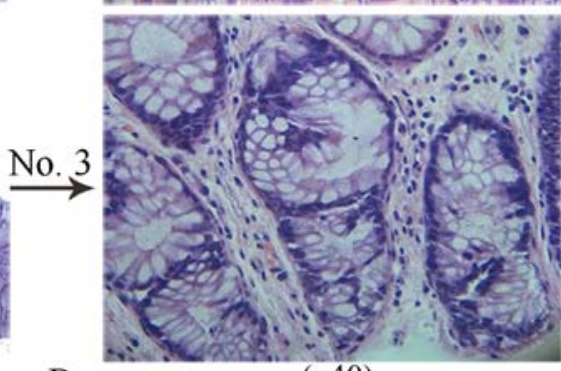

B

(x40)

Figure 1. (A) Schematic diagram for collecting a sample in this study and histologically altered tissue structure presented by H\&E staining. (B) After staining, cytoplasm presents faint red, and nuclei are bluish violet. The ocular lens used in this study was $\mathrm{x} 10$ ).

kit (Exiqon), and finally dried by centrifugation for $5 \mathrm{~min}$ at $400 \mathrm{rpm}$. Then the slides were scanned using the Axon GenePix 4000B microarray scanner (Axon Instruments). Scanned images were then imported into GenePix Pro 6.0 software (Axon Instruments) for grid alignment and data extraction. Replicated miRNAs were averaged and miRNAs with intensities $\geq 30$ in all samples were chosen for calculating the normalization factor. Expressed data were normalized using the median normalization. After normalization, for quality assessment of miRNA data, box plot, correlation matrix and scatter plot for miRNAs were used, significant differentially expressed miRNAs were identified through volcano plot filtering (fold-change $\geq 1.5$, P-value $\leq 0.05$ ). Hierarchical clustering was performed using MEV software (v4.6, TIGR). Quality control was by NanoDrop 1000 spectrophotometer and standard denaturing agarose gel electrophoresis.

qRT-PCR validation assay for target miRNAs. Total RNA was isolated and abstracted using TRIzol. Random primer $10 \mu \mathrm{mol} / \mathrm{l}$ was added to total RNA $(1 \mu \mathrm{g})$ from samples, then diethylpyrocarbonate was blended for $10 \mathrm{~min}$, finally, reverse transcription reaction liquids (5X RT reaction buffer $5 \mu 1$, dNTP $1 \mathrm{mmol} / 1$, RNase inhibitor $1 \mathrm{U} / \mu 1$, M-MLV RTase $8 \mathrm{U} / \mu \mathrm{l}$, diethylpyrocarbonate $4 \mu \mathrm{l}$ ) were added for $1 \mathrm{~h}$ to obtain reverse transcription products. SYBR-Green I was used to obtain quantitative RT-PCR according to manufacturer's instructions, relative expression levels were calculated by subtracting the average U6 (internal control) $\mathrm{Ct}$ from the $\mathrm{Ct}$ of the target gene obtained from the same cDNA and applying the formula $2^{-\Delta \Delta \mathrm{Ct}}$. All samples were in triplicate. The primers used by Primer 5.0 ABI Prism 7900 system are listed in Table I. A 2X qRT-PCR reaction mix $8 \mu \mathrm{l}$ (2X Master mix $5 \mu \mathrm{l}$, PCR sequence specific primer F $0.5 \mu \mathrm{l}$, PCR sequence specific primer R $0.5 \mu \mathrm{l}$ ) was added to holes corresponding to 384-PCR plate, then corresponding $2 \mu \mathrm{l}$ cDNA was added. Three-step program was used for the PCR reaction, after the reaction, analysis of melting curves was performed.

Statistic analysis. All data analysis was performed by SPSS 17.0 software. The RT-PCR results are presented as 
Table I. The primers used for qRT-PCR.

\begin{tabular}{|c|c|c|c|}
\hline Gene name & RT two-way primer & $\begin{array}{c}\text { Annealing } \\
\text { temperature }\left({ }^{\circ} \mathrm{C}\right)\end{array}$ & $\begin{array}{l}\text { Length of } \\
\text { products (bp) }\end{array}$ \\
\hline \multirow[t]{2}{*}{ U6 } & F: 5'GCTTCGGCAGCACATATACTAAAAT3' & & \\
\hline & R: 5'CGCTTCACGAATTTGCGTGTCAT3' & 60 & 89 \\
\hline \multirow[t]{2}{*}{ hsa-miR-429 } & GSP: 5'GGGGGTAATACTGTCTGGT3' & & \\
\hline & R: 5'TGCGTGTCGTGGAGTC3' & 60 & 64 \\
\hline \multirow[t]{2}{*}{ hsa-miR-200a-5p } & GSP: 5'GGGCATCTTACCGGACAG3' & & \\
\hline & R: 5'CAGTGCGTGTCGTGGAGT3' & 60 & 65 \\
\hline \multirow[t]{2}{*}{ hsa-miR-185-5p } & GSP: 5'GGTGGAGAGAAAGGCAGT3' & & \\
\hline & R: 5'TGCGTGTCGTGGAGTC3' & 60 & 59 \\
\hline \multirow[t]{2}{*}{ hsa-miR-29c-3p } & GSP: 5'GGGGTAGCACCATTTGAA3' & & \\
\hline & R: 5'CAGTGCGTGTCGTGGAG3' & 60 & 66 \\
\hline \multirow[t]{2}{*}{ hsa-miR-1237 } & GSP: 5'GGGTCCTTCTGCTCCGTC3' & & \\
\hline & R: 5'GTGCGTGTCGTGGAGTCG3' & 60 & 62 \\
\hline \multirow[t]{2}{*}{ hsa-miR-625-3p } & GSP: 5'GCGGCAGACTATAGAACTTT3' & & \\
\hline & R: 5'CAGTGCGTGTCGTGGA3' & 60 & 68 \\
\hline \multirow[t]{2}{*}{ hsa-miR-497-5p } & GSP: 5'GGGCAGCAGCACACTGT3' & & \\
\hline & R: 5'CAGTGCGTGTCGTGGAGT3' & 60 & 64 \\
\hline
\end{tabular}

GSP is a specific primer corresponding to miRNAs and R is a primer corresponding to RT primer.

the mean \pm SEM. One-way ANOVA analysis was used to accomplish the comparison among three groups, if the data were normal distribution, otherwise, non-parametric test was performed. Significant level $\alpha$ was 0.05 , and P-values $<0.05$ were considered to be statistically significant. All experiments were independently performed a triplicate.

\section{Results}

Histologically varied tissue structures are present in different tissue samples. Normal tissue structure, an important element of microenvironment, is responsible for stabilization of internal milieu. Stable tissue microenvironment exactly mediates generation and release of growth signaling responsible for cell proliferation and differentiation to sustain architecture and function of normal tissue (16). Alteration of tissue architecture and homeostasis is an early signal initiating carcinogenesis $(17,18)$. As for large intestine, crypts are structurally fundamental element of epithelial tissues and functionally basic unit for performing the above biological activities. In this context, we explored biological characteristics during colorectal cancerization on the basis of the crypt. To obtain histologically legible stucture, we performed HE staining. As shown in Fig. 1B, regular crypt structure is shown in no. 1, lumens of gland and monolayer cell nuclei in crypt were normal in dimension. However, crypt structure in no. 2 presented irregular shapes showing bigger and zigzag lumens of gland, and elongated cell nuclei with crowded and multilayer arrangement, hence, these crypts are called aberrant crypt foci (ACF) which is consist of a certain number of accumulated aberrant crypts with or without atypical hyperplasia (19). Significantly enlarged crypts in dimension are demonstrated in no. 3 whose closest distance to the $\mathrm{CRC}$ lesions is about $2.0 \mathrm{~cm}$, the aberrant crypts revealed that these nuclei were larger than those of normal cells, the arrangement of the cells was more crowded and multilayered, and polarity of cells had disappeared, which suggest that histologically dramatic alteration appears in colorectal mucosa in the process of canceration.

Changed biological hallmarks exist in different tissues. Tumor microenvironment (TME), a contributor of carcinogenesis, includes not only tumor tissues within TME, but also other tissues and stoma cells and large groups of cytokines produced by the cells. Therefore, in addtion to the exploration of histological alteration in colon epithelium, study of several stroma cells within tissue microenvironment was also performed by our group.

Cyclin D1 (CD1), a marker of tumor cells and a mediator of cell cycle, can regulate transformation of G1/S phase. However, excessive cell division can be caused by overexpressed CD1 through regulation of cell cycle resulting in uncontrolled cell growth, leading to formation of neoplasm. Thus, many studies have demonstrated $\mathrm{CD} 1$ overexpression in various forms of cancer (20-22). CD133, a member of cytomembrane protein superfamily, is deemed as a marker of tumor stem cells of CRC $(23,24)$. Increasing tumor cells and stem cells during colorectal cancerization can be presented by the expression of CD1 and CD133. Cytoskeleton, a kind of dominant tissue structure, is responsible for supporting cell stucture and accommodating components in cells, and associated with cell behavior, signal pathway, and apoptosis (25), and alteration of cytoskeleton can directly contribute to oncogenesis. Cytokeratin 18 (CK18), a component of epithelial cytoskeleton, served as a specific biomarker for the epithelium (26). Vimentin, 


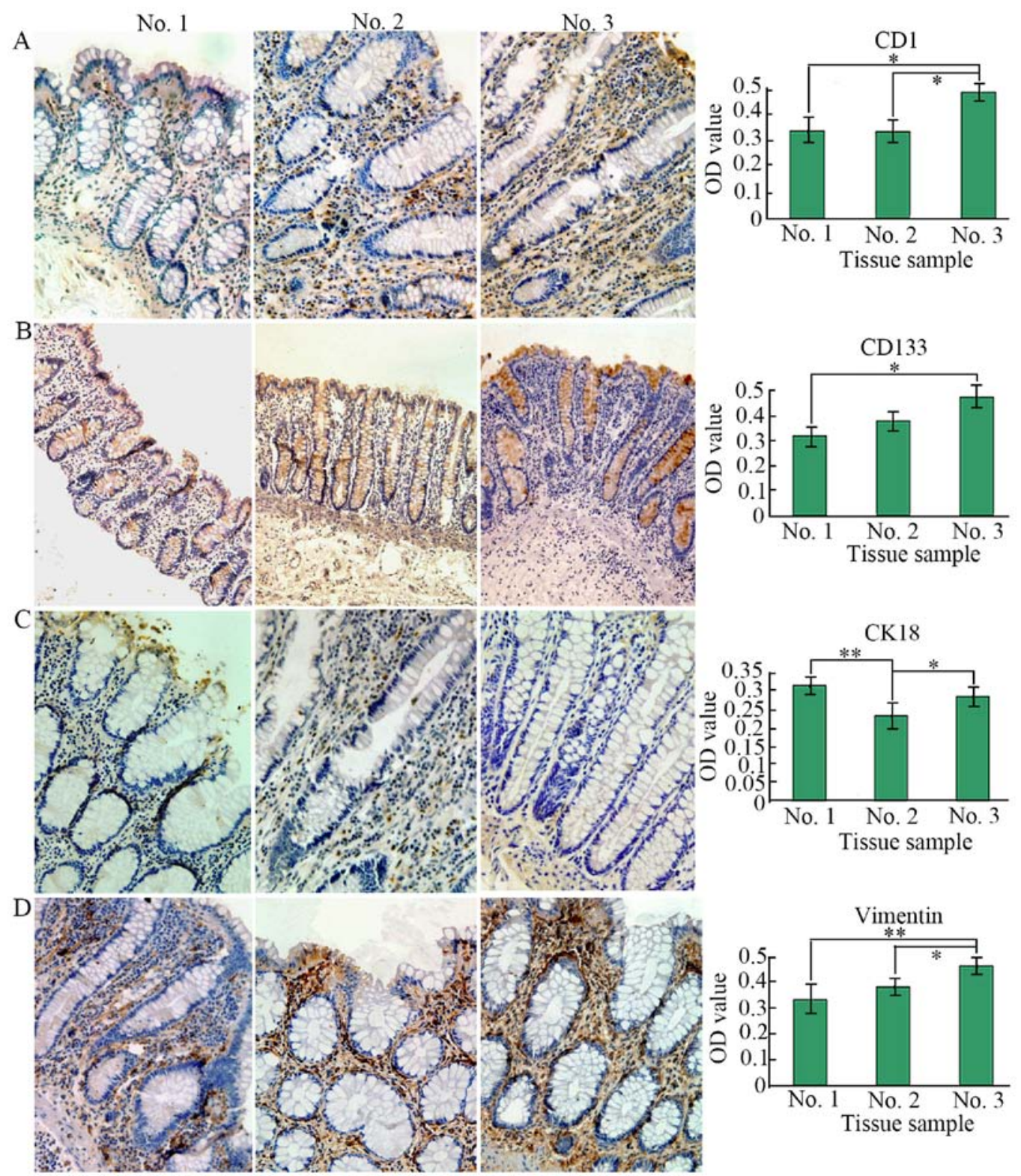

Figure 2. CK18, CD1, CD133, and vimentin immunohistochemical expression in different tissue samples. (A-D) The expression of CK18, CD1, CD133, and vimentin, respectively. ${ }^{* *} \mathrm{P}<0.01$ and ${ }^{*} \mathrm{P}<0.05$, respectively; magnification, $\mathrm{x} 400$ ).

a kind of intermediate filament, is also an important epithelial cytoskeleton taking part in various biological functions including cell growth, differentiation, signal transduction, and regulation of interaction between cytoskeleton proteins and cell adhesion molecules (27). In addition, its expression is located in mesenchyme and hence acted as marker of fibroblasts. Therefore, CD133, CD1, CK18, and vimentin were selected in our study.

To analyze the expression level of the four biological makers, we performed IHC staining. In this study, as shown in Fig. 2, we found that the expression of these markers was dysregulated, for instance, CK18 had a high expression in no. 1 whose microenvironment may be normal, namely, its expression was higher than that of no. $2(\mathrm{P}<0.01)$ and no. 3 $(\mathrm{P}>0.05)$, showing alteration of cytokeleton in no. 3 , while its corresponding mesenchymal marker vimentin revealed a low expression in no. 1, but its upregulation was found in no. 3 and in mesenchyme of crypts whose microenviron- ment is likely to be abnormal and tumorigenic responsible for colorectal carcinogenesis, thus leading to high expression of CD133 and CD1 which were higher than that of no. $1(\mathrm{P}<0.05)$. CD133 expression was mainly in the interior of crypts instead of the mesenchyme. On the contrary, CD1 expression was mainly in mesenchyme between crypts instead of in crypts. Hence, the results demonstrated increased tumor stem cells and their more extensive distribution. Besides, no. 2 represents a kind of transitional tissue which is between normal and cancerous, hence presenting an inconclusive variation.

Expression of DNMTs is significantly upregulated in para-carcinoma tissues. To examine DNMT expression under different tissue microenvironment, we respectively carried out IHC staining and western blotting for protein quantification. Our results, as demonstrated in Fig. 3A, revealed that expression of DNMT1, DNMT3A, and DNMT3B was 


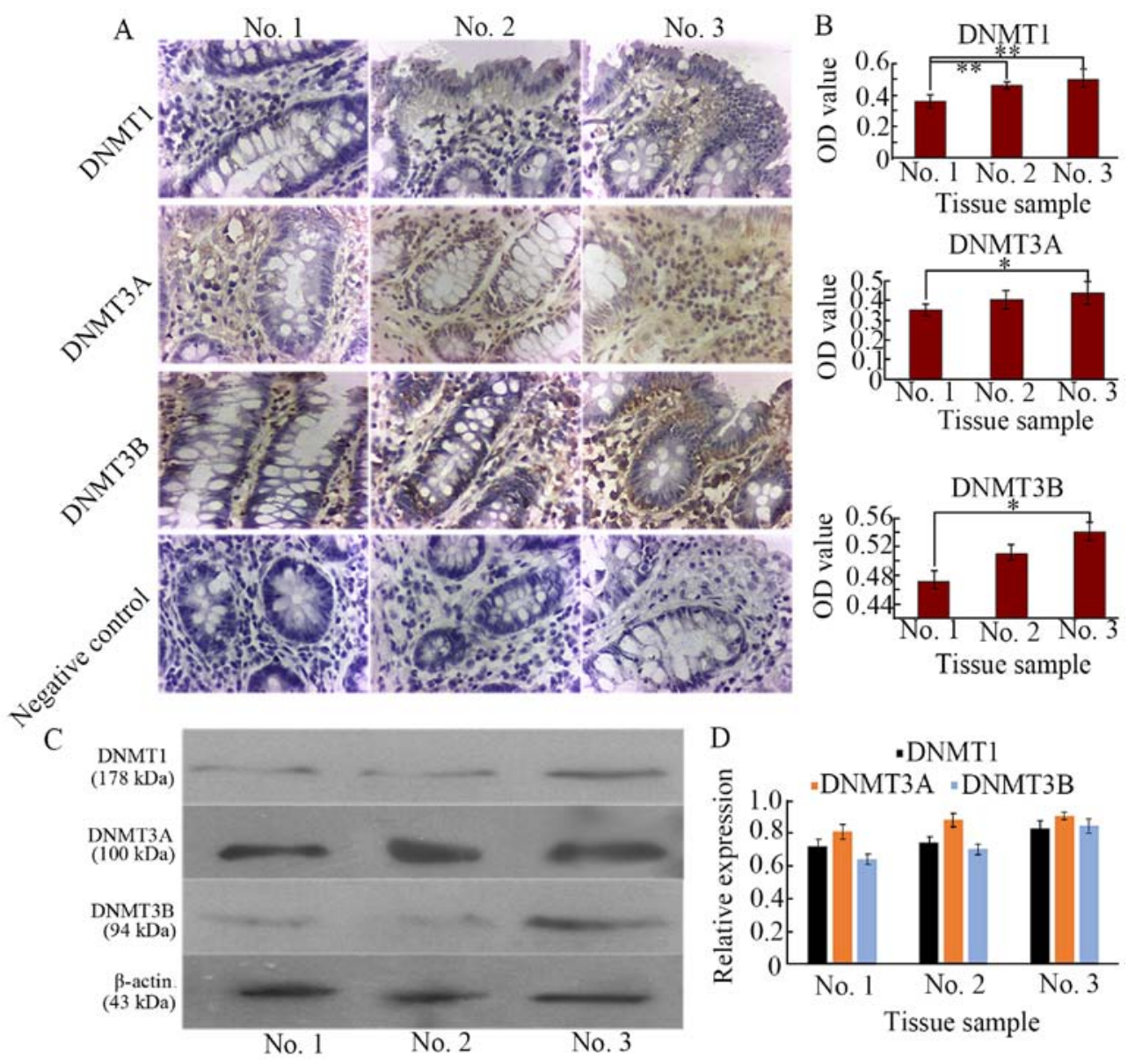

Figure 3. (A and B) Immunohistochemical staining and (C and D) western blotting of DNA methyltransferases (DNMTs) in different tissue samples. Negative expression of DNMT1, DNMT3A and DNMT3B in negative control ( ${ }^{* *} \mathrm{P}<0.01$ and ${ }^{*} \mathrm{P}<0.05$, respectively; magnification, $\left.\mathrm{x} 400\right)$.
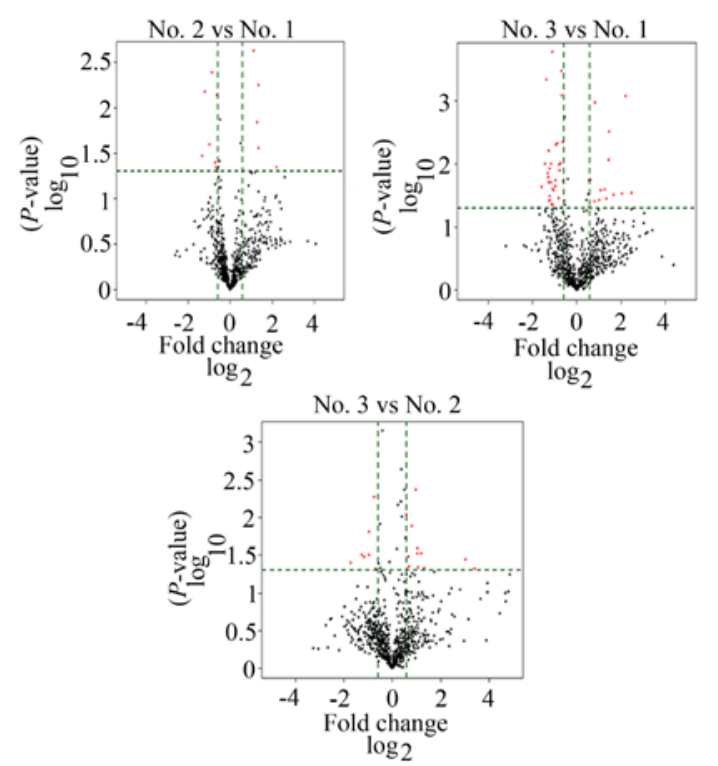

Figure 4. Volcano plot of differentially expressed miRNAs (the vertical lines correspond to 1.5 -fold up and down, respectively, and the horizontal line represents a P-value of 0.05 . The red point in the plot represents the differentially expressed miRNAs with statistical significance).

mainly found in aberrant tissues (no. 3), and weak staining was observed in normal tissue (no. 1), implying that alteration of DNA methylation existed in the aberrant tissues leading to abnormal DNA methylation within the promoter regions of genes, consequently, inactivated tumor suppressor gene (TSG) or other related genes including CK18 and reactiveted oncogenes including CD1, vimentin, and CD133 in tumor cells are caused, which are considered to be the most well-defined epigenetic signature in cancer $(28,29)$. All negative controls revealed negligible background staining. These DNMT levels were significantly higher compared with those in the no. 1 $(\mathrm{P}<0.05)$ shown by OD value (Fig. 3B). The higher the degree of dysplasia, the higher the expression level of DNMTs, DNMT1 expression mainly existed in mesenchyme between crypts, but in no. 2 , its expression was more and located in both mesenchyme and crypts. High expressed DNMT3A in no. 2 and no. 3 was mainly in mesenchyme, while high DNMT3B expression was in both mesenchyme and crypts (Fig. 3A). To further acquire protein quantification, western blotting demonstrated by Fig. 3C and D revealed the expression profile of the DNMTs similar to IHC results. Substantial research has shown that DNA methylation appears in the cancerous and para-cancerous tissues (30), these data are in agreement with our present study.

Distinguishable miRNA expression profiling in tissue samples. To screen differentially significantly expressed miRNAs in no. 1 , no. 2 and no. 3 , we performed a miRNA microarray. After volcano plot filtering (Fig. 4), the differentially significant miRNAs were displayed. As shown in Fig. 5, no. 2 


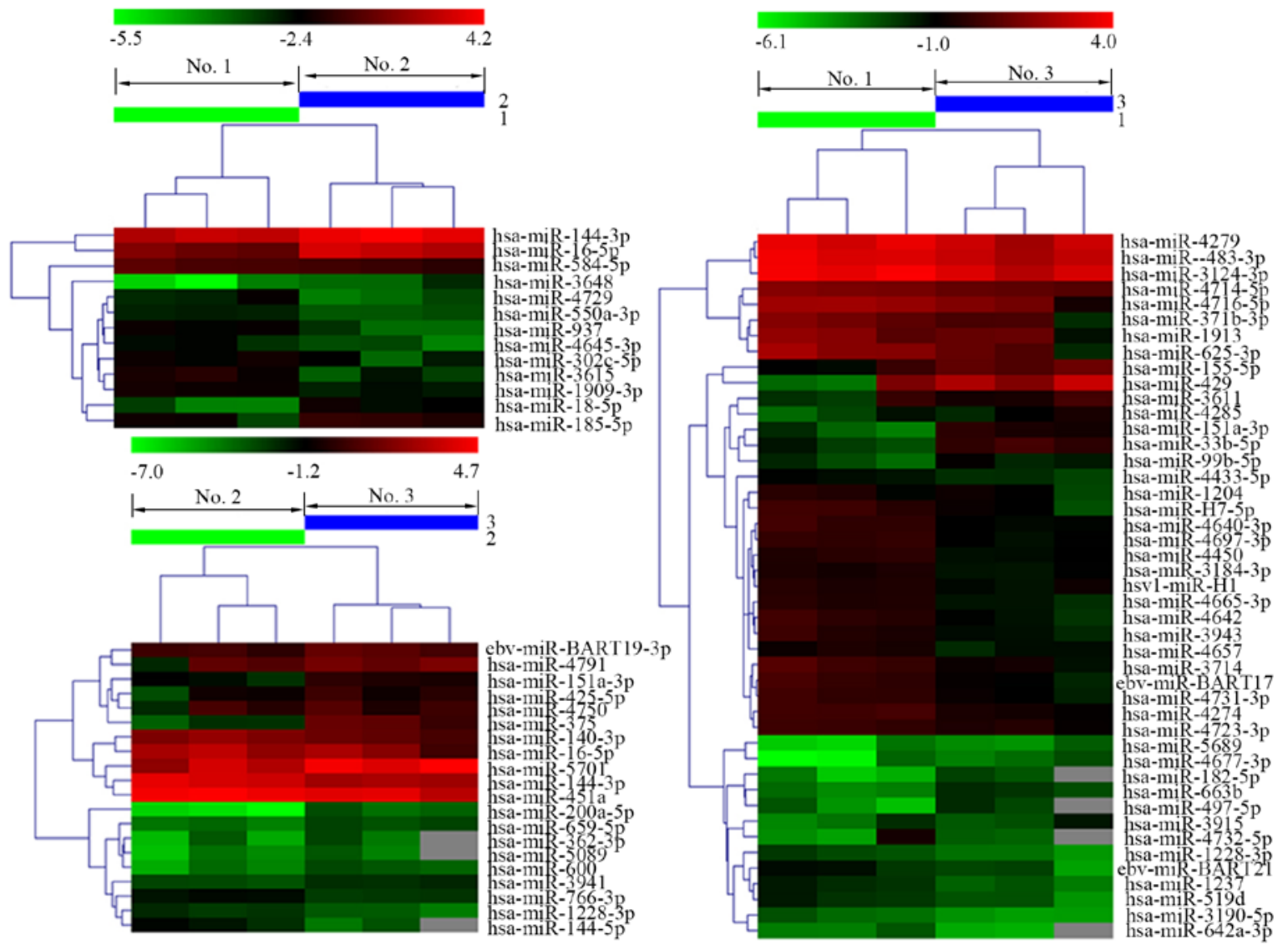

Figure 5. Heatmap and hierarchical clustering of differentially expressed miRNAs (the heatmap diagram shows the result of the two-way hierarchical clustering of miRNAs and samples. Each row represents a miRNA and each column represents a sample. The miRNA clustering tree is shown on the left, and the sample clustering tree appears on the top. Cluster analysis arranges samples and miRNAs into groups based on their fold-change of expression levels, which allows us to hypothesize about the relationships between miRNAs and samples. The color of each pattern indicates the fold change from green to red as from -5.5 to $4.2,-6.1$ to 4.0 and 7.0 to 4.7 , respectively).

vs. no. 1,13 miRNAs were filtered, among them, 5 miRNAs were upregulated, 8 miRNAs were downregulated. No. 3 vs. no. 1,45 miRNAs were differently expressed, 13 miRNAs were upregulated, 32 miRNAs were downregulated. No. 3 vs. no. 2, 13 miRNAs were upregulated, 7 miRNAs were downregulated. Previous studies have discovered that the degree of epigenetic difference between cancer cells and normal cells was considerably higher than that of normal cells with different phenotypes and even different germ cells, such as fibroblasts and epithelial cells (2). In this study, we found a cluster of upregulated and downregulated miRNAs in no. 2 and no. 3 tissues compared to no. 1 with normal microenvironment, implying differences of DNA methylation pattern in different tissues. These results reveal that cancer cells are different from normal cells in epigenetics, consistent with the previous studies. In addition, complexity and frequency of epigenetic alterations observed in cancer cells appear to be able to overturn the explanation that epigenetic alterations rely on single event (2), on the contrary, these pathological alterations may be caused by multiple mechanisms. Our results included in total 78 dramatically expressed miRNAs, and many upregulated or downregulated miRNAs also emerged in the same tissues, implying that some co-regulatory mechanisms may exist between different miRNAs or the same miRNAs may be co-regulated by several distinctive mechanisms.
Screening miRNAs targeting DNMTs and $q R T-P C R$ validation of the target miRNAs. Four main DNMT isoforms are present in mammalian cells. DNMT1, the maintenance methyltransferase, is responsible for the conservation of methyl marks during DNA replication, while, DNMT3A and DNMT3B are contributed mainly to de novo DNA methylation, DNMT1, the catalytically inactive isoform, has a synergy with DNMT3A and DNMT3B (31), although a study appears to disagree with this rigid classification (32). These DNMTs play an important role in DNA methylation pattern of cellular processes, for example, differentiation of stem cells during embryonic development $(33,34)$. However, abnormal DNA methylation profile caused by DNMT mutations or overexpression is likely to be an early epigenetic event during oncogenesis (33). Other epigenetic regulators including miRNAs are also involved in aberrant mediation of DNA methylation profile, and their combination participates in tumor transformation (35-38). Our results found the aberrant changes of DNMTs in the precancerous lesions of CRC, revealing abnormal accumulation of methylated DNA.

To determine the miRNAs targeting DNMTs, we adopted predictive information from the following three databases, mirbase, miranda and targetscan (http://www.ebi.ac.uk/ enright-srv/microcosm/htdocs/targets/v5/, http://www. microrna.org/microrna/home.do, http://mir-db.org/miRDB/), and overlapping parts of the three predictive results served as 
Table II. Average $2^{-\Delta \Delta \mathrm{Ct}}$ of 6 target miRNAs.

Tissue samples

\begin{tabular}{lccc}
\cline { 2 - 4 } Genes (DNMTs targeted) & No. 1 & No. 2 & No. 3 \\
\hline hsa-miR-185-5p (DNMT1) & $0.893 \pm 0.298$ & $2.310 \pm 0.259$ & $0.848 \pm 0.146$ \\
hsa-miR-200a-5p (DNMT3B) & $1.147 \pm 0.1934$ & $1.272 \pm 0.0769$ & $2.301 \pm 1.0458$ \\
hsa-miR-429 (DNMT3A) & $1.104 \pm 0.162$ & $0.151 \pm 0.014^{\mathrm{b}}$ & $2.241 \pm 0.211$ \\
hsa-miR-497-5p (DNMT3B) & $0.611 \pm 0.114^{\mathrm{a}}$ & $1.486 \pm 0.101$ & $0.966 \pm 0.082$ \\
hsa-miR-625-3p (DNMT3B) & $0.513 \pm 0.141$ & $0.339 \pm 0.115$ & $0.125 \pm 0.009$ \\
hsa-miR-1237 (DNMT3A) & $0.647 \pm 0.103$ & $0.348 \pm 0.039$ & $0.314 \pm 0.022$ \\
U6 (internal control) & $12.479 \pm 0.112$ & $12.605 \pm 0.161$ & $12.209 \pm 0.256$ \\
\hline
\end{tabular}

${ }^{\mathrm{a} N o .} 2$ vs. no. $3, \mathrm{P}<0.05 .{ }^{\mathrm{b}}$ No. 2 vs. no. $1, \mathrm{P}<0.05$.

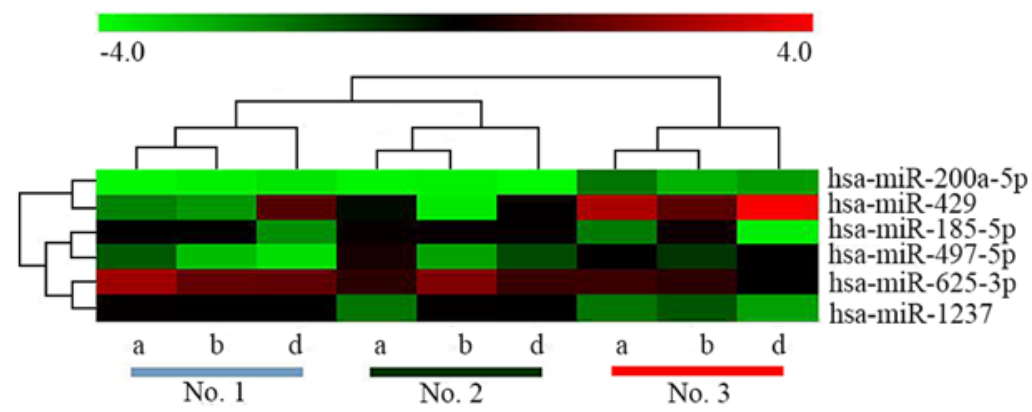

Figure 6. Heatmap and hierarchical clustering of six target miRNAs ( $\mathrm{a}, \mathrm{b}$ and $\mathrm{d}$ is the number of sample. The miRNA clustering tree is shown on the left, and the sample clustering tree appears on the top. The color of each pattern indicates the fold-change as $\log _{2}\left(2^{-\Delta \Delta \mathrm{Ct}}\right)$ from green to red from -4.0 to 4.0 . As seen from the clustering relationship, hsa-mir-200a-5p and hsa-miR-429 may have similar function.

the final result prediction. As a result, three target miRNAs were screened by the database, and other three target miRNAs were obtained through previous literature. Finally, six target miRNAs including hsa-miR-185-5p, hsa-miR-1237, hsa-miR-429, hsa-miR-200a-5p, hsa-miR-497-5p, and hsa-miR-625-3p were selected. These miRNAs target DNMT1, DNMT3A, and DNMT3B (as shown in Table II). Because of better sample clustering after removing $\mathrm{c}$ and e (c and e represent number of each sample), hierarchical clustering of six target miRNAs for 3 samples of every location was performed (Fig. 6). As shown in Fig. 6 and Table II, expression level of hsa-miR-200a-5p and hsa-miR-429 in no. 3 was higher than that of no. 1 and no. 2 (P-value of hsa-miR-429<0.05), indicating that low expressed DNA methylation profile probably participates in the formation of certain oncology mechanisms. For example, global DNA hypomethylation mainly occurs in dinucleotide of cytosine guanine $(\mathrm{CpG})$ within mobility and repetitive genome components, including satellite DNA sequence (pericentromeric regions), repetitive sequence of long interspersed nuclear element (LINE) and retrotransposon (39). Hypomethylation profile results in destruction of normal dinucleotide sequence and damage of chromosome stability through accumulating chromosome damage and inducing the reactivation of previously silenced genetic elements (retrotransposon), leading to the formation of various forms of cancer (40). A study has reported that miR-429 can target phosphatase and tensin homolog (PTEN) in endometrial cancer as a TSG and contribute to oncogenesis (41). Furthermore, in addition to genomic instability resulted from DNA hypomethylation, positive transcriptional regulation of proto-oncogenes is also a stimulative process of tumor initiation $(42,43)$. Recently, studies have found that mutation of TSG adenomatous polyposis coli (APC) is a driving event in initiation of coloretal cancer and seems to control expression and activity of DNMTs, then leading to demethylation of numerous genes and forcing intestinal epithelial cells to maintain a undifferentiated and progenitor-like status (44). Similar to APC mutation, whether the aberrant miRNAs observed in our study are involved in this mechanism needs to be thoroughly investigated in the future.

Furthermore, DNMTs mediated by miRNAs play an important role in hypermethylation of $\mathrm{CpG}$ islands. Downregulated hsa-miR-185-5p, hsa-miR-497-5p, hsa-miR-625-3p and hsa-miR-1237 were observed in no. 3 tissue, implying increased level of DNMTs. These results suggest that $\mathrm{CpG}$ islands have an inclination to hypermethylation, because $\mathrm{CpG}$ islands of colon epithelial cells are not methylated under normal conditions $(35,45)$, therefore, $\mathrm{CpG}$ island methylation pattern in tissue cells with inclination to canceration have altered. For example, the expression levels of hsa-miR-625-3p and hsa-miR-1237 had a decreasing tendency from normal tissues to precancerous lesions, although their difference was not statistically significant. While the expression levels of hsamiR-185-5p and hsa-miR-497-5p of no. 2 location were more than that of no. 1 (hsa-miR-185-5p had no statistical differ- 
ence), implying that possibly some mechanisms are involved in these changes where miR-185-5p and miR-497-5p of no. 2 tissue were upregulated.

\section{Discussion}

Under general conditions, clinical and pathological studies have found that direct infiltration range of colon cancer along the distance at both ends is no more than $2 \mathrm{~cm}$, while the distance that lymph nodes around the colon can spread bilaterally exceeds $8 \mathrm{~cm}$. In our samples, the distance of no. 1 to CRC lesions is more than $10 \mathrm{~cm}$. The no. 1 deemed as safe location of surgery resection is regarded as normal tissue. On the contrary, the distance of no. 3 from CRC lesions is no more than $2 \mathrm{~cm}$, which is regarded as para-carcinoma tissue. Tissue of no. 2 is between no. 1 and no. 3 .

Accumulating evidence suggests that neoplasms are complex tissues consisted of multiple different cell types participating in heterotypic interactions with one another. During the past decade, an emerging notion that the contributions of the TME to carcinogenesis must be considered in the biology of neoplasms (16), revealing an important role of local tissue microenvironment involving in the formation of tumor-associated stroma in tumorigenesis. To date, several studies have found that altered tissue microenvironment has an effect on colorectal carcinogenesis, and epigenetic changes function as a key player in cellular response to the alteration of microenvironment (46-48), which are consistent with our results revealing the histologically altered morphologies of tissues with different distance to CRC lesions, loss of normal crypts and existence of many ACF in no. 3, however, tissues no. 2 have shown inclination to canceration under altered microenvironment. Abundance of oncogene CD1 and CD133 expression appears in no. 3 where the aberrant tissue structures are formed, indicating the establishment of tumor-associated microenvironment which provides support and shield for growth of tumor cells (49). On the contrary, our data revealed that significantly decreased CK18 expression and increased vimentin expression were found in no. 3, indicating an emergence of altered cytoskeleton in the process of colorectal cancerization, which is in agreement with the epithelial-mesenchymal transition (EMT) defined by Thompson et al (50).

Epigenetic alterations in cancer are mediated by complex network of co-factors, such as DNA methylases, histone modification enzymes and non-coding RNAs (51). DNA methylation can fight against activation of reverse transcription transposon and promote stability of chromosomes and then become genome guardian (33). Functional DNA methylation patterns in normal cells are frequently in disorder, presenting global genome hypomethylation and local hypermethylation of TSGs. Global hypomethylation is associated with genome instability, including probable onco-gene activation, while hypomethylation of promoter will often lead to the aberrant silence of TSGs (52). Chromatin structure changes with global hypomethylation will result in increased instability of genome, which can be a key motivator of colon canceration (53). For example, the decreased methylation levels of microsatellite DNA sequences [simple sequence repeats $(\mathrm{SSR})]$ easily lead to gene mutation, which has been validated in many tumor models (52). Previous studies have shown that aberrant DNA methylation and miRNA alteration may play a vital role in different steps of cancer formation (54). Therefore, our study explored expression of DNMT and miRNA profiling in colon tissues with different location, and assessed the relative levels of miRNAs targeting corresponding DNMTs by quantitative real-time PCR. In our study, the downregulation of hsa-miR-185-5p, hsa-miR497-5p, hsa-miR-625-3p and hsa-miR-1237 in canceration tissues (no. 3) suggests that aberrant DNMT expression has led to DNA hypermethylation pattern demonstrated by high expressed DNMT1, DNMT3A, and DNMT3B in no. 3 (Fig. 3), which is likely to prompt increased instability of chromosome structure and overexpression of some oncogenes. The difference of miRNA profiling in the different phases of colorectal canceration reveals that oncogenesis is not a molecular event but a sequential process involving a number of genes and signal molecules. Furthermore, two or more miRNAs may take part in the canceration process at the same time. For example, hsa-miR-200a-5p and hsa-miR-429 present upregulated tendency from no. 1 to no. 3 , although the difference of miR-200a-5p has no statistical significance, showing a viewpoint that they have a synergistic function during colorectal carcinogenesis.

In conclusion, the results presented for the first time show histological alteration in different colorectal tissue microenvironment using colorectal tissue samples with diverse distance from CRC lesions. Besides, highly expressed DNMT1, DNMT3A, DNMT3B and distinguishable miRNA expression profiling in cancerous tissues compared with the histopathologically unchanged indicated the pivotal function of DNA methylation and miRNAs in initiation of CRC under altered tissue microenvironment. Although DNA methylation level and miRNAs are considered as a single biomarker for early cancer detection, other epigenetic biomarkers, such as histone modifications, circulating cell-free DNA and long non-coding RNA, may have more potential. A panel of DNA methylations of miRNAs, epigenetic hallmarks, can have potential in the early detection of CRC and screening patients with high risk factors.

\section{Acknowledgements}

This study was supported by the National Science Foundation of China (no. 81173257).

\section{References}

1. Jemal A, Bray F, Center MM, Ferlay J, Ward E and Forman D: Global cancer statistics. CA Cancer J Clin 61: 69-90, 2011.

2. Dawson MA and Kouzarides T: Cancer epigenetics: From mechanism to therapy. Cell 150: 12-27, 2012.

3. Migheli F and Migliore L: Epigenetics of colorectal cancer. Clin Genet 81: 312-318, 2012.

4. Feinberg AP and Tycko B: The history of cancer epigenetics. Nat Rev Cancer 4: 143-153, 2004.

5. Sahnane N, Magnoli F, Bernasconi B, Tibiletti MG, Romualdi C, Pedroni M, Ponz de Leon M, Magnani G, Reggiani-Bonetti L, Bertario L, et al; AIFEG: Aberrant DNA methylation profiles of inherited and sporadic colorectal cancer. Clin Epigenetics 7: 131,2015

6. Baylin SB, Herman JG, Graff JR, Vertino PM and Issa JP: Alterations in DNA methylation: A fundamental aspect of neoplasia. Adv Cancer Res 72: 141-196, 1998. 
7. Robertson KD, Uzvolgyi E, Liang G, Talmadge C, Sumegi J, Gonzales FA and Jones PA: The human DNA methyltransferases (DNMTs) 1, 3a and 3b: Coordinate mRNA expression in normal tissues and overexpression in tumors. Nucleic Acids Res 27: 2291-2298, 1999

8. Stefani G and Slack FJ: Small non-coding RNAs in animal development. Nat Rev Mol Cell Biol 9: 219-230, 2008.

9. Hwang HW and Mendell JT: MicroRNAs in cell proliferation, cell death, and tumorigenesis. Br J Cancer 94: 776-780, 2006.

10. Rangel-Salazar R, Wickström-Lindholm M, Aguilar-Salinas CA, Alvarado-Caudillo Y, Døssing KB, Esteller M, Labourier E, Lund G, Nielsen FC, Rodríguez-Ríos D, et al: Human native lipoprotein-induced de novo DNA methylation is associated with repression of inflammatory genes in THP-1 macrophages. BMC Genomics 12: 582, 2011.

11. Mori Y, Ishiguro H, Kuwabara Y, Kimura M, Mitsui A, Ogawa R, Katada T, Harata K, Tanaka T, Shiozaki M, et al: MicroRNA-21 induces cell proliferation and invasion in esophageal squamous cell carcinoma. Mol Med Rep 2: 235-239, 2009.

12. Iorio MV, Ferracin M, Liu CG, Veronese A, Spizzo R, Sabbioni S, Magri E, Pedriali M, Fabbri M, Campiglio M, et al: MicroRNA gene expression deregulation in human breast cancer. Cancer Res 65: 7065-7070, 2005.

13. Song YX, Yue ZY, Wang ZN, Xu YY, Luo Y, Xu HM, Zhang X, Jiang L, Xing CZ and Zhang Y: MicroRNA-148b is frequently down-regulated in gastric cancer and acts as a tumor suppressor by inhibiting cell proliferation. Mol Cancer 10: 1, 2011.

14. Matsubara N: Epigenetic regulation and colorectal cancer. Dis Colon Rectum 55: 96-104, 2012.

15. Wu WK, Law PT, Lee CW, Cho CH, Fan D, Wu K, Yu J and Sung JJ: MicroRNA in colorectal cancer: From benchtop to bedside. Carcinogenesis 32: 247-253, 2011.

16. Hanahan D and Weinberg RA: Hallmarks of cancer: The next generation. Cell 144: 646-674, 2011.

17. Egeblad M, Nakasone ES and Werb Z: Tumors as organs: Complex tissues that interface with the entire organism. Dev Cell 18: 884-901, 2010.

18. Weber CE and Kuo PC: The tumor microenvironment. Surg Oncol 21: 172-177, 2012.

19. Paulsen JE, Namork E, Steffensen IL, Eide TJ and Alexander J: Identification and quantification of aberrant crypt foci in the colon of Min mice - a murine model of familial adenomatous polyposis. Scand J Gastroenterol 35: 534-539, 2000.

20. Serrano M, Hannon GJ and Beach D: A new regulatory motif in cell-cycle control causing specific inhibition of cyclin D/CDK4. Nature 366: 704-707, 1993.

21. Lin RJ, Lubpairee T, Liu KY, Anderson DW, Durham S and Poh CF: Cyclin D1 overexpression is associated with poor prognosis in oropharyngeal cancer. J Otolaryngol Head Neck Surg 42: 23, 2013.

22. Ren B, Li W, Yang Y and Wu S: The impact of cyclin D1 overexpression on the prognosis of bladder cancer: A meta-analysis. World J Surg Oncol 12: 55, 2014.

23. O'Brien CA, Pollett A, Gallinger S and Dick JE: A human colon cancer cell capable of initiating tumour growth in immunodeficient mice. Nature 445: 106-110, 2007.

24. Ricci-Vitiani L, Lombardi DG, Pilozzi E, Biffoni M, Todaro M, Peschle C and De Maria R: Identification and expansion of human colon-cancer-initiating cells. Nature 445: 111-115, 2007.

25. Snásel J, Shoeman R, Horejsí M, Hrusková-Heidingsfeldová O, Sedlácek J, Ruml T and Pichová I: Cleavage of vimentin by different retroviral proteases. Arch Biochem Biophys 377: 241-245, 2000.

26. Hondo T, Kanaya T, Takakura I, Watanabe H, Takahashi $\mathrm{Y}$, Nagasawa Y, Terada S, Ohwada S, Watanabe K, Kitazawa H, et al: Cytokeratin 18 is a specific marker of bovine intestinal M cell. Am J Physiol Gastrointest Liver Physiol 300: G442-G453, 2011.

27. Singh S, Sadacharan S, Su S, Belldegrun A, Persad S and Singh G: Overexpression of vimentin: Role in the invasive phenotype in an androgen-independent model of prostate cancer. Cancer Res 63: 2306-2311, 2003.

28. Previati M, Manfrini M, Galasso M, Zerbinati C, Palatini J, Gasparini P and Volinia S: Next generation analysis of breast cancer genomes for precision medicine. Cancer Lett 339: 1-7, 2013.

29. Chen XY, He QY and Guo MZ: XAF1 is frequently methylated in human esophageal cancer. World J Gastroenterol 18: 2844-2849, 2012.
30. Cao XY, Ma HX, Shang YH, Jin MS, Kong F, Jia ZF, Cao DH, Wang YP, Suo J and Jiang J: DNA methyltransferase3a expression is an independent poor prognostic indicator in gastric cancer. World J Gastroenterol 20: 8201-8208, 2014.

31. Seidel C, Florean C, Schnekenburger M, Dicato M and Diederich M: Chromatin-modifying agents in anti-cancer therapy. Biochimie 94: 2264-2279, 2012.

32. Walton EL, Francastel C and Velasco G: Maintenance of DNA methylation: Dnmt3b joins the dance. Epigenetics 6: 1373-1377, 2011.

33. Van Emburgh BO and Robertson KD: Modulation of Dnmt3b function in vitro by interactions with Dnmt3L, Dnmt3a and Dnmt3b splice variants. Nucleic Acids Res 39: 4984-5002, 2011.

34. Ooi SK, O'Donnell AH and Bestor TH: Mammalian cytosine methylation at a glance. J Cell Sci 122: 2787-2791, 2009.

35. Schnekenburger $M$ and Diederich M: Epigenetics Offer New Horizons for Colorectal Cancer Prevention. Curr Colorectal Cancer Rep 8: 66-81, 2012.

36. Florean C, Schnekenburger M, Grandjenette C, Dicato M and Diederich M: Epigenomics of leukemia: From mechanisms to therapeutic applications. Epigenomics 3: 581-609, 2011.

37. Kanai Y and Hirohashi S: Alterations of DNA methylation associated with abnormalities of DNA methyltransferases in human cancers during transition from a precancerous to a malignant state. Carcinogenesis 28: 2434-2442, 2007.

38. Karius T, Schnekenburger M, Ghelfi J, Walter J, Dicato M and Diederich M: Reversible epigenetic fingerprint-mediated glutathione-S-transferase P1 gene silencing in human leukemia cell lines. Biochem Pharmacol 81: 1329-1342, 2011.

39. van Engeland M, Derks S, Smits KM, Meijer GA and Herman JG: Colorectal cancer epigenetics: Complex simplicity. J Clin Oncol 29: 1382-1391, 2011.

40. Vaiopoulos AG, Athanasoula KC and Papavassiliou AG: Epigenetic modifications in colorectal cancer: Molecular insights and therapeutic challenges. Biochim Biophys Acta 1842: 971-980, 2014.

41. Yoneyama K, Ishibashi O, Kawase R, Kurose K and Takeshita T: miR-200a, miR-200b and miR-429 are onco-miRs that target the PTEN gene in endometrioid endometrial carcinoma. Anticancer Res 35: 1401-1410, 2015.

42. Roy S and Majumdar AP: Cancer stem cells in colorectal cancer: genetic and epigenetic changes. J Stem Cell Res Ther (Suppl 7): pii: 10342

43. Tost J: DNA methylation: An introduction to the biology and the disease-associated changes of a promising biomarker. Mol Biotechnol 44: 71-81, 2010.

44. Hammoud SS, Cairns BR and Jones DA: Epigenetic regulation of colon cancer and intestinal stem cells. Curr Opin Cell Biol 25: 177-183, 2013.

45. Choong MK and Tsafnat G: Genetic and epigenetic biomarkers of colorectal cancer. Clin Gastroenterol Hepatol 10: 9-15, 2012.

46. Mima K, Nishihara R, Qian ZR, Cao Y, Sukawa Y, Nowak JA, Yang J, Dou R, Masugi Y, Song M, et al: Fusobacterium nucleatum in colorectal carcinoma tissue and patient prognosis. Gut: Aug 26, 2015 (Epub ahead of print). doi: 10.1136/gutjnl-2015-310101

47. Tahara T, Yamamoto E, Suzuki H, Maruyama R, Chung W, Garriga J, Jelinek J, Yamano HO, Sugai $\mathrm{T}$, An B, et al: Fusobacterium in colonic flora and molecular features of colorectal carcinoma. Cancer Res 74: 1311-1318, 2014.

48. Ogino S, Lochhead P, Chan AT, Nishihara R, Cho E, Wolpin BM, Meyerhardt JA, Meissner A, Schernhammer ES, Fuchs CS, et al: Molecular pathological epidemiology of epigenetics: Emerging integrative science to analyze environment, host, and disease. Mod Pathol 26: 465-484, 2013.

49. Potter JD: Morphogens, morphostats, microarchitecture and malignancy. Nat Rev Cancer 7: 464-474, 2007.

50. Thompson EW, Newgreen DF and Tarin D: Carcinoma invasion and metastasis: A role for epithelial-mesenchymal transition? Cancer Res 65: 5991-5995, discussion 5995, 2005.

51. González-Ramírez I, Soto-Reyes E, Sánchez-Pérez Y,Herrera LA and García-Cuellar C: Histones and long non-coding RNAs: The new insights of epigenetic deregulation involved in oral cancer. Oral Oncol 50: 691-695, 2014.

52. Robertson KD: DNA methylation and human disease. Nat Rev Genet 6: 597-610, 2005.

53. Li J, Jin H and Wang X: Epigenetic biomarkers: potential applications in gastrointestinal cancers. ISRN Gastroenterology 2014: 464015, 2014

54. Calin GA and Croce CM: MicroRNA signatures in human cancers. Nat Rev Cancer 6: 857-866, 2006. 